Turbulence modeling for Francis turbine water passages simulation

This article has been downloaded from IOPscience. Please scroll down to see the full text article.

2010 IOP Conf. Ser.: Earth Environ. Sci. 12012070

(http://iopscience.iop.org/1755-1315/12/1/012070)

View the table of contents for this issue, or go to the journal homepage for more

Download details:

IP Address: 193.226.8.90

The article was downloaded on 03/09/2010 at 07:10

Please note that terms and conditions apply. 


\title{
Turbulence modeling for Francis Turbine water passages simulation
}

\author{
P Maruzewski ${ }^{1}$, H Hayashi ${ }^{2}$, C Munch ${ }^{1}$, K Yamaishi ${ }^{2}$, T Hashii ${ }^{2}$, H P Mombelli ${ }^{1}$, \\ Y Sugow $^{2}$ and F Avellan ${ }^{1}$ \\ ${ }^{1}$ Ecole polytechnique fédérale de Lausanne, Laboratory of Hydraulic Machines \\ Avenue de Cour 33 bis, CH-1007 Lausanne, Switzerland \\ ${ }^{2}$ Nippon KOEI Power Systems, 1-22 Doukyu, Aza, Morijyuku, Sukagawa, Fukushima \\ Pref. 962-8508, Japan \\ E-mail : pierre.maruzewski@epfl.ch
}

\begin{abstract}
The applications of Computational Fluid Dynamics, CFD, to hydraulic machines life require the ability to handle turbulent flows and to take into account the effects of turbulence on the mean flow. Nowadays, Direct Numerical Simulation, DNS, is still not a good candidate for hydraulic machines simulations due to an expensive computational time consuming. Large Eddy Simulation, LES, even, is of the same category of DNS, could be an alternative whereby only the small scale turbulent fluctuations are modeled and the larger scale fluctuations are computed directly. Nevertheless, the Reynolds-Averaged Navier-Stokes, RANS, model have become the widespread standard base for numerous hydraulic machine design procedures. However, for many applications involving wall-bounded flows and attached boundary layers, various hybrid combinations of LES and RANS are being considered, such as Detached Eddy Simulation, DES, whereby the RANS approximation is kept in the regions where the boundary layers are attached to the solid walls. Furthermore, the accuracy of CFD simulations is highly dependent on the grid quality, in terms of grid uniformity in complex configurations. Moreover any successful structured and unstructured CFD codes have to offer a wide range to the variety of classic RANS model to hybrid complex model. The aim of this study is to compare the behavior of turbulent simulations for both structured and unstructured grids topology with two different CFD codes which used the same Francis turbine. Hence, the study is intended to outline the encountered discrepancy for predicting the wake of turbine blades by using either the standard k- $\varepsilon$ model, or the standard k- $\varepsilon$ model or the SST shear stress model in a steady CFD simulation. Finally, comparisons are made with experimental data from the EPFL Laboratory for Hydraulic Machines reduced scale model measurements.
\end{abstract}

\section{Introduction}

For engineering applications, Reynolds' averaging method is based on separating variables into average and fluctuating parts. When the averaging operation is applied to the equations of motion, one obtains the well-known RANS equations that control the average flow field. The effect of turbulent fluctuations appearing in the Reynolds stress terms must be modeled to close the system. The solution of the RANS equations allows predicting the flow in fairly complex configurations.

A very wide range of models for the Reynolds stress is available, ranging from simple algebraic models, $k-\varepsilon$ models to full algebraic Reynolds-stress closures.

The one-equation model is solving a transport equation either for the turbulent kinetic energy or directly for the eddy viscosity. Spalart and Allmaras have proposed an improved one-equation model [1] based on an iterative procedure for developing the transport equation adapted to the increasing complexity of the flow.

The two-equation models are the more popular since they offer a good compromise between versatility and computing effort. The typical model examples are the $k-\varepsilon$ model proposed by Launder in 1974; see [2] and $k-\omega$ model developed twenty years later by Wilcox [3]. A comprehensive and critical review of closure methods for two-equation turbulence models can be found in [3]. Using a combination of singular perturbation methods and numerical computations, the conventional $k-\varepsilon$ formulations are found to be inaccurate for boundary layers in 
adverse pressure gradient; the use of wall function tends to overcome the shortcomings of such a model. In 1994, Menter [4] proposed the shear stress transport, SST model, mixing the $k$ - $\omega$ model close to walls and the k-e model for the main flow far away from the walls. Since the early $1990 \mathrm{~s}$, both the tremendous computing power increase and the development of those two-equation models enable the systematic use of the flow numerical simulations for investigating complex configurations.

The full algebraic Reynolds-stress model is solving the transport equations for the Reynolds stress tensor. The eddy viscosity assumption is no longer used enabling to take into account the turbulence anisotropy. In such a model, the system of RANS equations is thus closed with six transport equations which make Reynolds stress model well suited to complex flows. However, the increased sophistication of the turbulence model makes the computing effort heavier than for the previous "simpler" RANS models.

The challenge is now to use the suitable numerical methods among DNS, RANS, LES and Hybrid methods, according to the type of investigated flow. In turbomachinery applications, the first simulation was performed by Raw et al [5] in 1989. A three-dimensional steady Reynolds Averaged Navier-Stokes RANS simulation was carried out with a standard $k-\varepsilon$ turbulence model and logarithmic wall functions. Then, Roach, ten years later, carried out a posteriori numerical error estimation to improve accuracy of the numerical simulations [6]. Various approaches to estimate error and quantify uncertainty are presented. Systematic grid-convergence studies constituted the most reliable technique for the quantification of numerical uncertainty. Commercial computational fluid software featuring incompressible flow solver for hydraulic turbines applications [7] became available since the early 1990's.

The scope of the present paper is to present the RANS simulation to predict the performance of a hydraulic machine as well as the wake of component blades. First the Nippon KOEI Power System Francis turbine reduced scale model is presented. The following section describes the computing domain, the grids and the turbulence models. In the last section, the numerical results are then presented through a sensitivity analysis as well as a local flow analysis.

\section{Case study: the Nippon KOEI Power System F44 turbine reduced scale model}

In the process of a turbine development, Nippon KOEI Power System, NKPS, was willing to investigate measurements and numerical simulations in a reduced scale model to predict the performances of their prototype. The NKPS-F44S turbine consisted of small head hydraulic Francis turbine. The case study was commissioned in 2008, see Fig. 1. This turbine had a rated power of $8.3 \mathrm{MW}$ under $105 \mathrm{~m}$ head.

EPFL was contracted by NKPS to manufacture and to test a reduced scale model homologous to the prototype. NKPS-F44 turbine model tests were performed at the EPFL Test Rig 2 in 2008. From the hill chart, the best efficiency operating point, called BEP, is chosen for this present analysis; see Table 1 [8].

Table 1: Operating point

\begin{tabular}{c|c|c|c|c}
\hline \hline $\begin{array}{c}\text { Operating } \\
\text { Point }\end{array}$ & Guide Vane Opening & $\begin{array}{c}\text { Specific Rotation } \\
\text { Speed }\end{array}$ & Specific Discharge & Head \\
\cline { 2 - 5 } & $d$ & $n_{11}$ & $Q_{11}$ & $H$ \\
\hline BEP & 0.02 & 60.4 & 0.55 & 30 \\
\hline
\end{tabular}

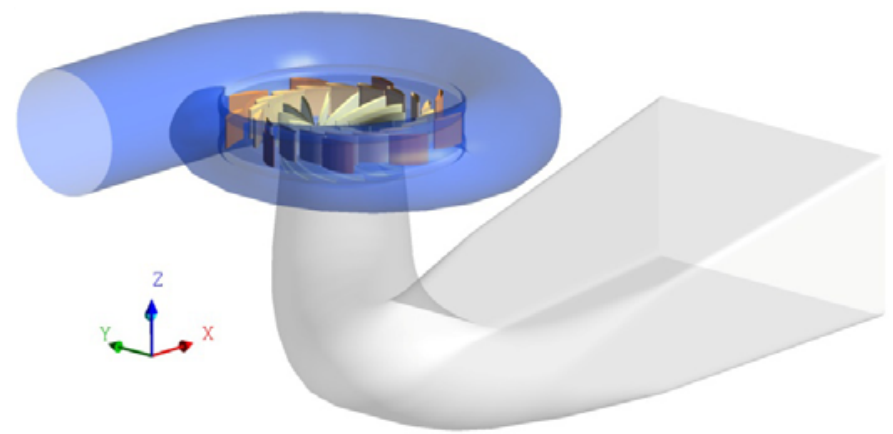

Fig. 1 NKPS-F44 Francis turbine reduced scale model 


\section{Numerical Setup}

\subsection{Computing Domain}

Volume and surfaces of the spiral casing, the stay vane, the guide vane and the draft tube are defined by NKPS drawings. The computing domain of NKPS-F44 reduced scale model built with previous volumes is represented in Fig. 1. The NKPS-F44 computing domain is defined by five volumes $\mathrm{V}$ and each component is identified with an index: $V_{s c}$ for the spiral casing volume, $V_{v}$ for the stay vane, $V_{o}$ for the guide vane, $V_{b}$ for the runner and $\mathrm{V}_{\mathrm{d}}$ for the draft tube.

\subsection{Grids}

All NKPS-F44 reduced scale model water passage components are properly meshed for generating a complete machine model. Two topologies of grid are applied: the first one is a structured topology and the second one an unstructured one. The properties of both topologies applied to the volume $\mathrm{V}_{\mathrm{sc}+\mathrm{v}+\mathrm{o}+\mathrm{b}+\mathrm{d}}$ are defined in Table 2 . The structured and unstructured grids are respectively presented in Fig. 2 and Fig. 3. The structured grid is generated by Ansys package and the unstructured grid by Star $\mathrm{CCM}+$ package.

Table 2: Properties of topologies of grid

\begin{tabular}{|c|c|c|c|c|c|c|c|c|c|c|}
\hline \multirow{2}{*}{ Topology } & \multicolumn{2}{|c|}{ Spiral casing } & \multicolumn{2}{|c|}{ Stay vane } & \multicolumn{2}{|c|}{ Guide vane } & \multicolumn{2}{|c|}{ Runner } & \multicolumn{2}{|c|}{ Draft tube } \\
\hline & Elements & Nodes & Elements & Nodes & Elements & Nodes & Elements & Nodes & Elements & Nodes \\
\hline Structured & 302,412 & 313,463 & 473,790 & 511,717 & 626,940 & 699,732 & $2,161,822$ & $2,289,560$ & 418,960 & 429,895 \\
\hline Unstructured & 924,344 & $4,551,746$ & 922,813 & $3,414,735$ & $1,075,699$ & $4,256,407$ & $1,645,548$ & $6,917,966$ & $1,335,284$ & $\begin{array}{c}6,295 \\
757\end{array}$ \\
\hline
\end{tabular}

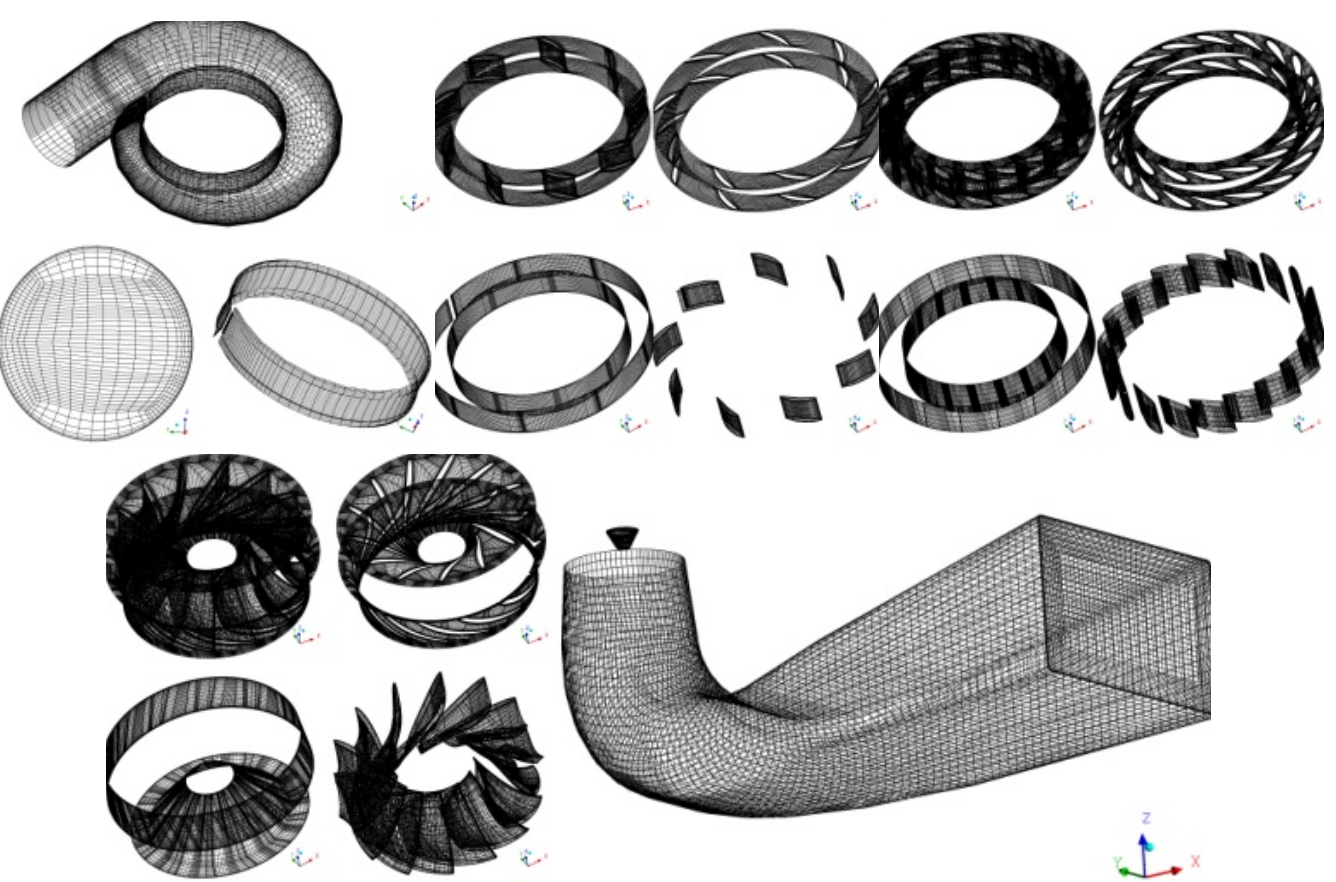

Fig. 2: Structured grids of the spiral casing, the stay vane, the guide vane, the runner and the draft tube
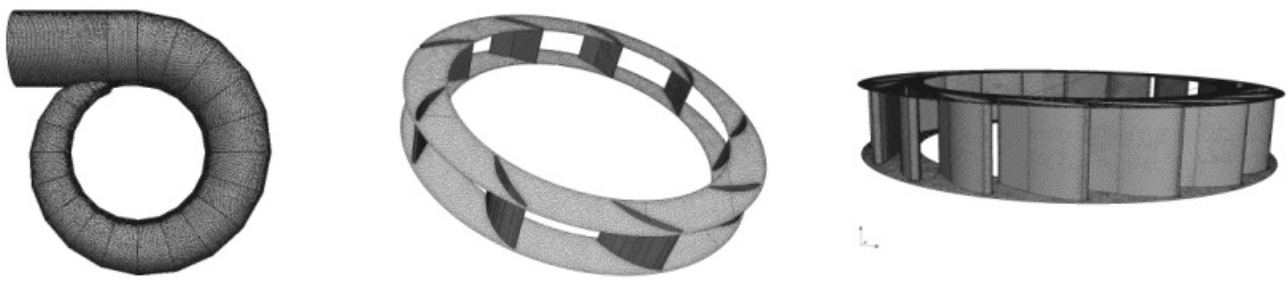

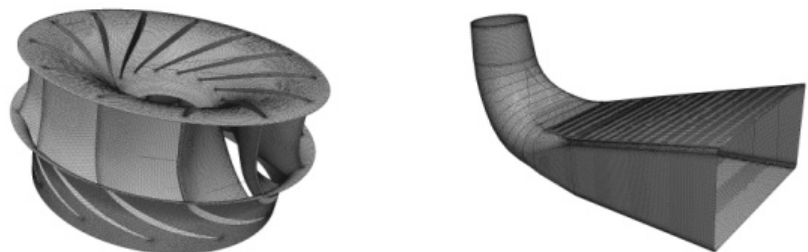

Fig. 3: Unstructured grids of the spiral casing, the stay vane, the guide vane, the runner and the draft tube

\subsection{Turbulence modeling}

An investigation was performed for identifying the turbulence models allowing accurate prediction of the wake of the guide vane blade and of the runner blade for both topologies of grids. Two-equation turbulence models are very widely used, as they offer a good compromise between numerical effort and computational accuracy. In this paper, three two-equation turbulence models were tested: the standard $k$ - $\varepsilon$ model, the standard $k$ $\omega$ model and the modified shear stress transport model SST.

The standard $k-\varepsilon$ model directly solves the differential transport equations for the turbulence kinetic energy $k$, and the turbulence dissipation rate $\varepsilon$ [2].

$$
\begin{aligned}
& \frac{\partial(\rho k)}{\partial t}+\nabla \cdot(\rho \vec{C} k)=\nabla \cdot\left[\left(\mu+\frac{\mu_{t}}{2}\right) \nabla k\right]-\rho \varepsilon \\
& \frac{\partial(\rho \varepsilon)}{\partial t}+\nabla \cdot(\rho \vec{C} \varepsilon)=\nabla \cdot\left[\left(\mu+\frac{\mu_{t}}{2}\right) \nabla \varepsilon\right]-1.92 \rho \frac{\varepsilon^{2}}{k}
\end{aligned}
$$

The standard $k$ - $\omega$ model directly solves the differential transport equations for the turbulence kinetic energy $k$ and, the turbulence frequency $\omega[3]$.

$$
\begin{aligned}
& \frac{\partial(\rho k)}{\partial t}+\nabla \cdot(\rho \vec{C} k)=\nabla \cdot\left[\left(\mu+\frac{\mu_{t}}{2}\right) \nabla k\right]-0.09 \rho k \omega \\
& \frac{\partial(\rho \omega)}{\partial t}+\nabla \cdot(\rho \vec{C} \omega)=\nabla \cdot\left[\left(\mu+\frac{\mu_{t}}{2}\right) \nabla \omega\right]-0.075 \rho \omega^{2}
\end{aligned}
$$

The Shear Stress Transport, SST, model combines the advantages of $k-\varepsilon$ and $k-\omega$ models for the transport of the turbulent shear stress and provides highly accurate predictions of the onset and the amount of flow separation under adverse pressure gradients. Furthermore, Menter's SST model contains a limit to the formulation of the eddy-viscosity [4].

Ansys CFX is used to perform turbulent simulation with the structured grid and Star CCM+ with the unstructured grid.

\section{Analysis and Discussion}

\subsection{Result sensitivities}

a) Grid sensitivity

Three structured grids are generated with different number of nodes. These grids are called coarse grid, medium grid and fine grid, see

Table 3. Hence, the mesh quality is evaluated by comparing the calculated pressure coefficient $\mathrm{cp}$ of coarse and medium grids to the one obtained for the fine mesh with SST model. The prediction error of $\mathrm{cp}$ versus the fine mesh is given in

Table 3. In the following sections, all following analysis is performed with the medium mesh with an error of $\pm 0.5 \%$ for predicting the $\mathrm{cp}$ value.

$$
c_{p}=\frac{p-p_{\text {inlet }}}{\rho\left(\frac{C_{m \overline{1} e}^{2}}{2}\right)}
$$

Table 3: Structured grid sensitivity 


\begin{tabular}{c|c|c}
\hline \hline $\begin{array}{c}\text { Mesh } \\
{[-]}\end{array}$ & $\begin{array}{c}\text { Number of nodes } \\
{[-]}\end{array}$ & $\begin{array}{c}\text { Error of } c_{p} \\
{[\%]}\end{array}$ \\
\hline Coarse & $1,306,284$ & 6.0 \\
\hline Medium & $4,195,944$ & 0.5 \\
\hline Fine & $11,740,091$ & 0.0 \\
\hline
\end{tabular}

b) Specific losses per component and efficiency between base line test and simulations

The specific losses per component depending on the turbulence model are evaluated and presented for the both topologies in Fig. 4 and Fig. 5. In the first hand, from topology differences, simulations with structured and unstructured grids reached the same level of total specific losses. In the second hand, from turbulence model approaches, the standard $k-\omega$ model seems to underestimate the specific losses in the runner compared to the results obtained with the standard $k-\varepsilon$ model. Furthermore, the SST model slightly predicts higher losses in the runner than using the other turbulence models.

A comparison between the efficiency measured during EPFL base line test, BLT, and the efficiency predicted by numerical simulations is performed for the different turbulence models, see

Table 4. All simulated efficiencies are in a good agreement with the measured value.

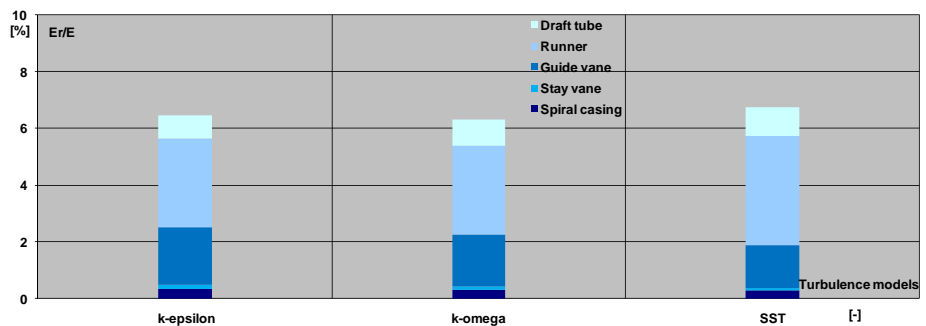

Fig. 4: Specific losses per component versus turbulence model for structured grids

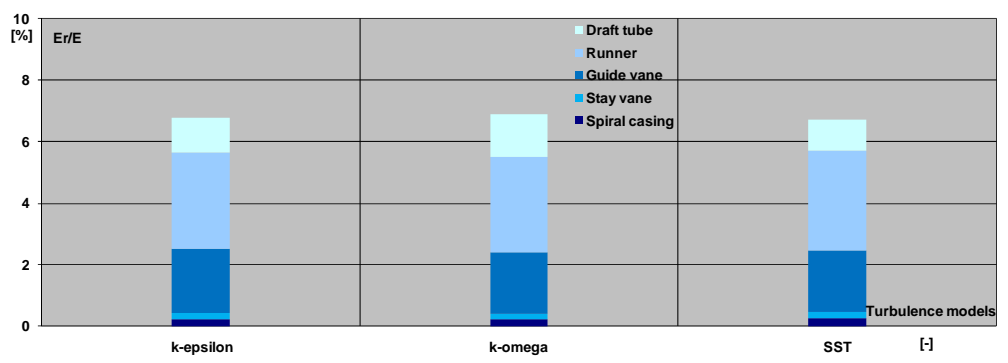

Fig. 5: Specific losses per component versus turbulence model for unstructured grids

Table 4: Efficiency comparisons

\begin{tabular}{|c|c|c|c|}
\hline $\begin{array}{c}\text { Test case } \\
{[-]}\end{array}$ & $\begin{array}{c}\text { BLT efficiency } \\
\frac{\boldsymbol{\eta}_{B L T}}{\boldsymbol{\eta}_{B L T}}[\%]\end{array}$ & $\begin{array}{l}\text { Numerical efficiency } \\
\qquad \frac{\boldsymbol{\eta}_{\text {mum }}}{\boldsymbol{\eta}_{B L T}}[\%]\end{array}$ & $\left.\begin{array}{c}\text { Difference } \\
\boldsymbol{\eta}_{B L T}-\boldsymbol{\eta}_{n u m} \\
\boldsymbol{\eta}_{B L T}\end{array} \%\right]$ \\
\hline Structured $k-\varepsilon$ & 100 & 100.3 & 0.30 \\
\hline Unstructured $k-\varepsilon$ & 100 & 100.0 & 0.00 \\
\hline Structured $k-\omega$ & 100 & 100.4 & 0.40 \\
\hline Unstructured $k-\omega$ & 100 & 99.8 & 0.14 \\
\hline Structured SST & 100 & 99.9 & 0.03 \\
\hline Unstructured SST & 100 & 100.0 & 0.05 \\
\hline
\end{tabular}

The small differences between the base line test efficiency values and numerical predicted specific hydraulic energy efficiency is among other reasons due to not taking into account neither the disk friction losses nor the volumetric losses in the numerical simulations.

\subsection{Wake behavior versus turbulence models}


The effect of the wake of the stay vane, and of the guide vane and the rotor-stator interaction between the runner and the guide vane for both topologies of grids with the standard $k$ - $\varepsilon$ turbulence model are presented by showing the isosurfaces of the vorticity at the mid plane of the stay vane, the guide vane and the runner in the Fig. 6. The simulations with both topologies reproduced the same wake of guide vane for fixed components of the turbine. But, however, a small discrepancy is noticed concerning the interaction of water with the leading edge of the runner. In the unstructured case, higher spot of vortices appeared in runner water passages near the leading edge of the runner. These spots could be an effect of the quality of the unstructured grid of the runner.

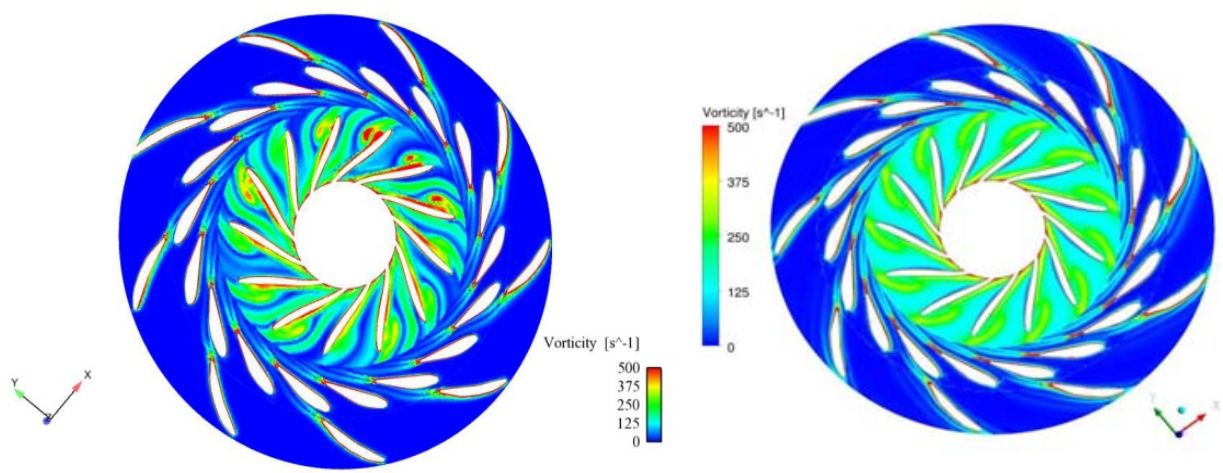

Fig. 6: Isosurfaces of the vorticity for both topologies of grids: unstructured on the left and structured on the right at mid plane of the stay vane, the guide vane and the runner

The Fig. 7 to the Fig. 10 showed the profiles of the velocity along a $360^{\circ}$ line at the mid plane of the spiral casing, the stay vane, the guide vane and the runner versus the different turbulence model.

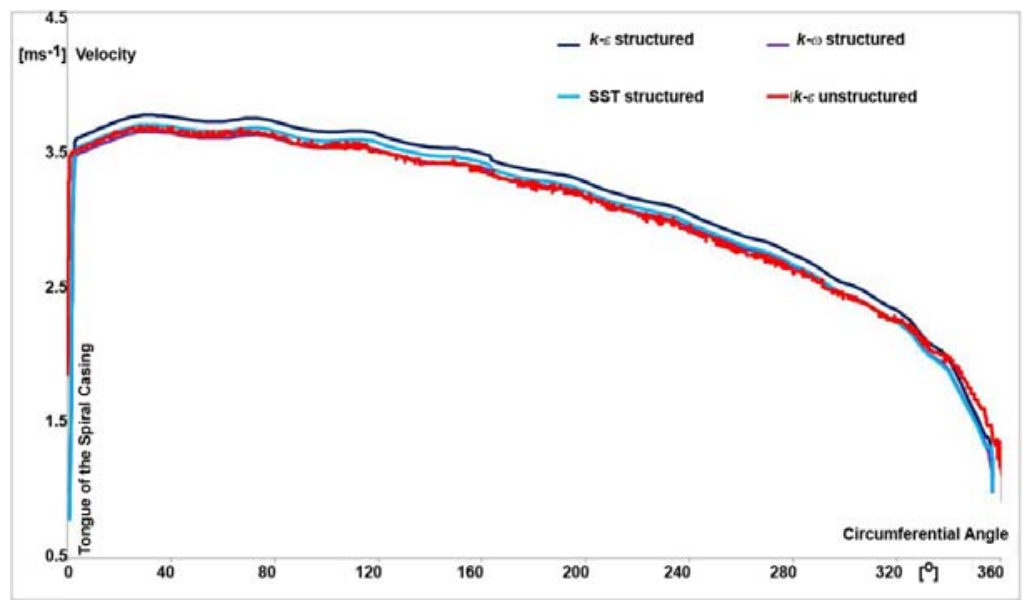

Fig. 7: Velocity profiles of the spiral casing at mid plane

In the spiral casing, Fig. 7, the standard $k-\varepsilon$ turbulence model for the structured grid overestimates the velocity whereas the standard $k$ - $\omega$ turbulence model underestimates the values. Then, the SST turbulence model becomes a good compromised turbulence model. Furthermore, the standard $k$ - $\varepsilon$ turbulence model for the unstructured grid gives the same profile as the standard $k$ - $\omega$ turbulence model for the structured grid except the area close to the tongue. 

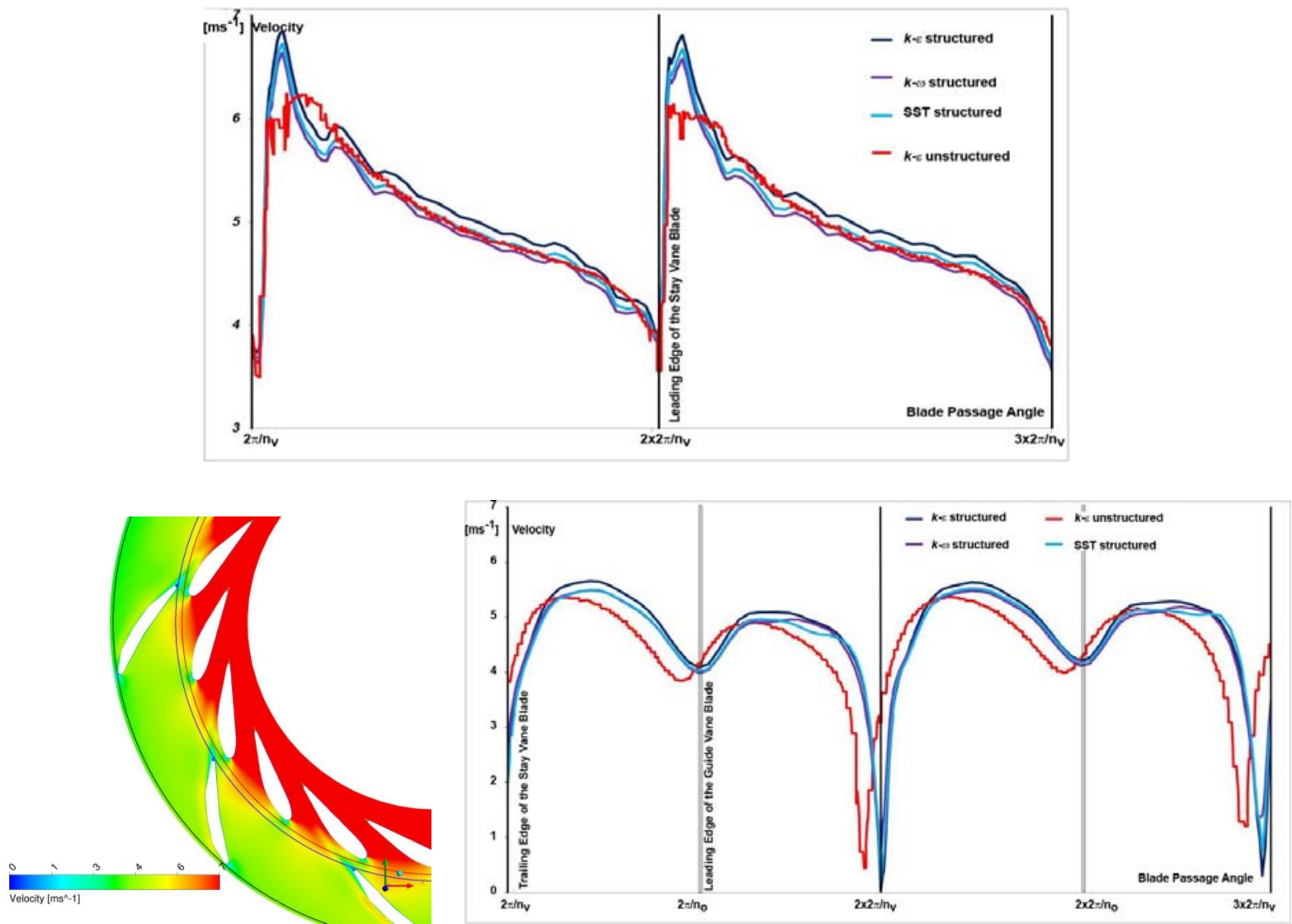

Fig. 8: Velocity profiles at mid plane of the stay vane: top) at the inlet and bottom) at the outlet

The profiles of the velocity at the inlet and at the outlet of the stay vane are represented in the Fig. 8 . The same trends about over- and underestimation for the structured grids are noticed with the standard $k-\varepsilon$ and $k-\omega$ turbulence models. But, whereas the SST turbulence model is still a good compromised between the both turbulence models at the inlet of the stay vane. It is observed that at the outlet of the stay vane, within the water passage and near the leading edge of the guide vane, the SST model is close to the standard $k$ - $\omega$ turbulence model, and close to the standard $k-\varepsilon$ turbulence model near the trailing edge of the stay vane. Furthermore, the standard $k-\varepsilon$ turbulence model for the unstructured grid underestimates the values of velocity.

The profiles of the velocity at the inlet and at the outlet of the guide vane are represented in the Fig. 9. Firstly, at the inlet of the guide vane, one water passage of the stay vane is linked with two water passages of the guide vane. The same behaviors of all three turbulence models are observed within two adjacent water passages of the guide vane as in the stay vane case. Nevertheless, all three turbulence models predict the same shape of the wake of the leading edge of the guide vane blade located in the middle of the water passage of the stay vane. Moreover, the standard $k$ - $\omega$ turbulence model overestimates the wake of the trailing edge of the stay vane and the profile of the velocity in the water passage delimited by the trailing edge of the stay vane and the leading edge of the guide vane. All three turbulence models simulate the same interaction between the wake of the trailing edge of the stay vane and the wake of the leading edge of the guide vane; see the small amplitude peak between the blade passage angles $\left[\frac{2 x 2 \pi}{n_{v}}: \frac{3 x 2 \pi}{n_{o}}\right]$ in the Fig. 9, top. Secondly, at the outlet of the guide vane two wakes of guide vanes blades appeared, see two peaks of velocity within the water passage of the guide vane in the Fig. 9, bottom. If, you fixed your attention to one guide vane blade, for instance, $\left[\frac{2 \times 2 \pi}{n_{o}}: \frac{3 \times 2 \pi}{n_{o}}\right]$ you understood that the smallest peak became from the wake of the previous guide vane blade $\frac{2 \times 2 \pi}{n_{o}}$ and the longest from the present guide 
vane $\frac{3 x 2 \pi}{n_{o}}$

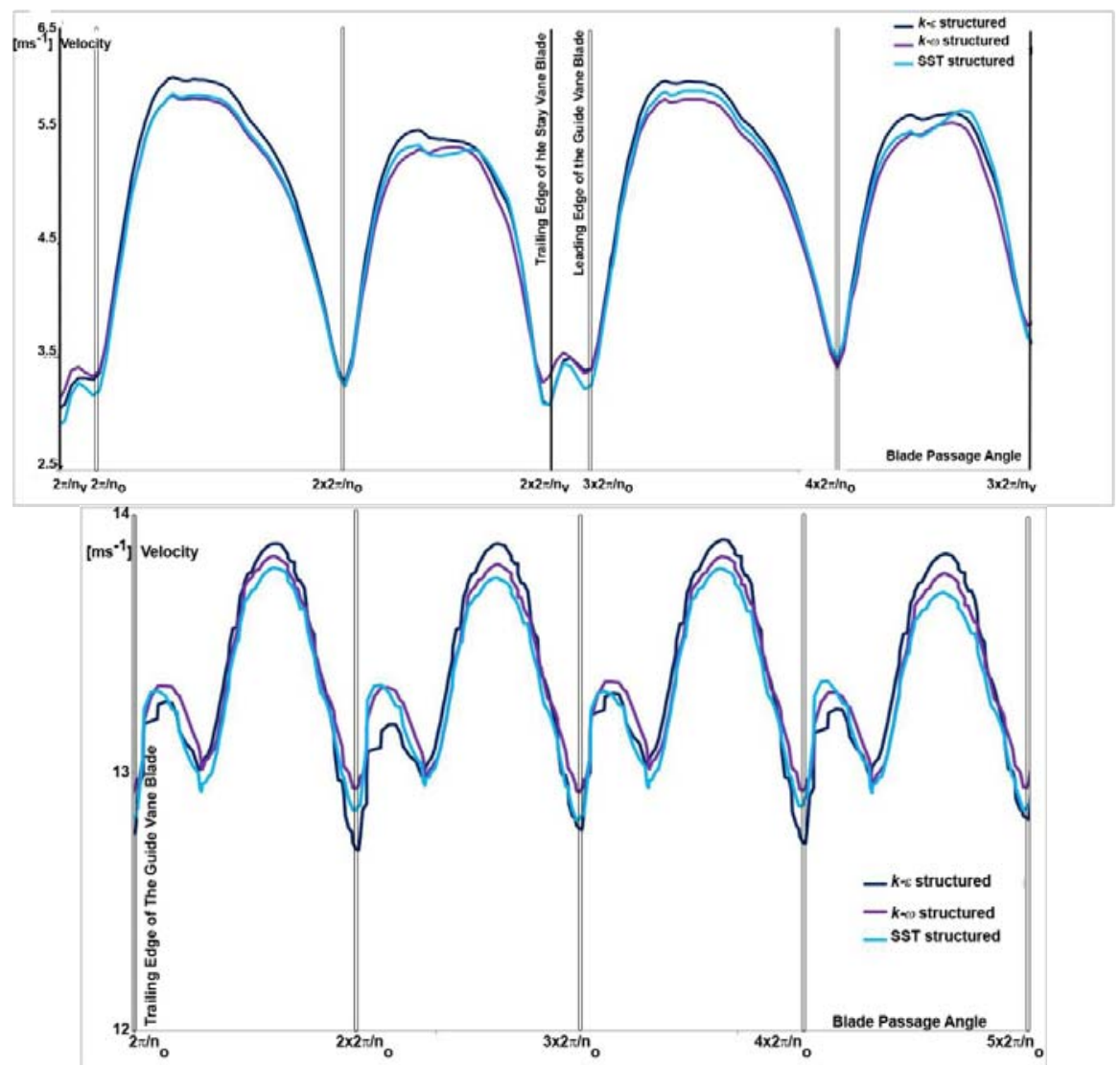

Fig. 9: Velocity profiles at mid plane of the guide vane: top) at the inlet and bottom) at the outlet The profiles of the velocity at the inlet of the runner are represented in the Fig. 10. All three turbulence models predict the same shape and amplitude of the wake of the leading edge of the runner blade.

\section{Conclusions}

The aim of this study was to compare the behavior of turbulent simulations for both structured and unstructured grids topology with two different CFD codes which used the same Francis turbine case. Hence, the study outlined the encountered discrepancy for predicting the wake of turbine blades by using either the standard $k-\varepsilon$ model, or the standard $k-\omega$ model or the SST shear stress model in a steady CFD simulation.

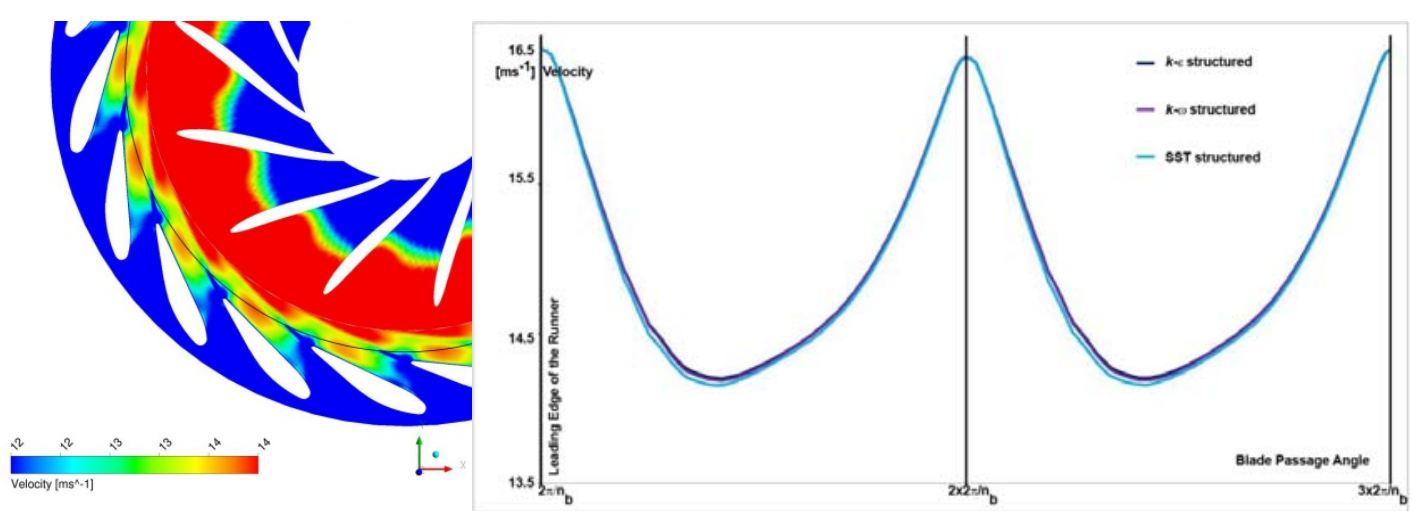

Fig. 10: Velocity profiles at mid plane of the runner at the inlet 
The simulations using unstructured grids and the standard $k-\varepsilon$ model underestimated the amplitude of the wake. However, both topologies, structured and structured grids, predicted the correct behavior of the wake. More specifically, all investigated turbulence models for structured grid reach the same level of accuracy for the prediction of the wake.

\section{Acknowledgments}

The authors are very grateful to Nippon KOEI Power System for its involvement and constant support to the project. Finally, the authors would like to express their sincere gratitude to the staff of the EPFL Laboratory for Hydraulic Machines, for their support and advice in contribution to this work.

\section{Nomenclature}

$\begin{array}{llll}C_{m} & \text { Meridional component of absolute velocity }\left[\mathrm{ms}^{-1}\right] & \varepsilon & \text { Turbulent dissipation rate }\left[\mathrm{m}^{2} \mathrm{~s}^{-3}\right] \\ c_{p} & \text { Pressure coefficient [-] } & \rho & \text { Density }\left[\mathrm{kg} \mathrm{m}^{3}\right] \\ d & \text { Guide vane opening [m] } & \eta & \text { Specific hydraulic efficiency [-] } \\ H & \text { Head [ m] } & \mu & \text { Dynamic viscosity }\left[\mathrm{kgm}^{-1} \mathrm{~s}^{-1}\right] \\ Q_{11} & \text { Specific discharge [-] } & \mu_{t} & \text { Turbulent viscosity }\left[\mathrm{kgm}^{-1} \mathrm{~s}^{-1}\right] \\ k & \text { Turbulent kinetic energy per unit mass [m2s-2] } & \omega & \text { Turbulent frequency }\left[\mathrm{s}^{-1}\right] \\ n_{11} & \text { Specific rotation velocity [-] } & & \\ n_{v}, n_{o}, n_{b} & \text { Number of stay vane, guide vane and runner blades } & & \\ p & \text { Static pressure [Pa] } & & \end{array}$

\section{References}

[1] Spalart P R and Allmaras S R 1994 A one-equation turbulence model for aerodynamic flows La Recherche Aérospatiale $15-21$

[2] Launder B E and Spalding D B 1974 The numerical computation of turbulent flows Comput. Methods in Appl. Mechs and Eng. 3(2) 269-89

[3] Wilcox D C 1993 Comparison of two-equation turbulence models for boundary layers with pressure gradient AIAA J. 31(8) 1414-21

[4] Menter F 1994 Two-equation eddy-viscosity turbulence model for engineering applications AIAA J. 30:6 1657-59

[5] Raw M J, Galpin P F and Hutchinson B R 1989 A Colocated Finite Volume Method for solving the NavierStokes Equations for Incompressible and Compressible Flows in Turbomachniery: Results and Applications Proc. of 2000 ASME Fl. Engin. Conf. (Boston, US, June 11-15)

[6] Roach P J 1997 Quantification of Uncertainty in Computational Fluid Dynamics Annu. Rev. Fluid Mech. 29 123-60

[7] AEA Technology Engineering Software Limited, 2000 CFX-TASCflow Theroy Documentation Version 2.10 (Waterloo, Ontario, Canada)

[8] EFPL NKPS-F44 Development project hydraulic performances Technical Report (Lausanne, 2009) 